\title{
G65.2+5.7: A Thermal Composite Supernova Remnant with a Cool Shell
}

\author{
R. L. Shelton ${ }^{1}$, K. D. Kuntz ${ }^{2}$, R. Petre ${ }^{3}$ \\ 1 The Department of Physics and Astronomy and the Center for Simulational Physics at \\ the University of Georgia, Athens, GA 30602 \\ ${ }^{2}$ The Department of Physics at the University University of Maryland at Baltimore County \\ ${ }^{3}$ NASA's Goddard Space Flight Center \\ rls@hal .physast.uga.edu \\ kuntz@milkyway.gsfc.nasa.gov \\ rob@milkyway.gsfc . nasa.gov
}

\begin{abstract}
This paper presents archival ROSAT PSPC observations of the G65.2+5.7 supernova remnant (also known as $G 65.3+5.7$ ). Little material obscures this remnant and so it was well observed, even at the softest end of ROSAT's bandpass $(\sim 0.11$ to $0.28 \mathrm{keV})$. These soft X-ray images reveal the remnant's centrally-filled morphology which, in combination with existing radio frequency observations, places G65.2+5.7 in the thermal composite (mixed morphology) class of supernova remnants. Not only might G65.2+5.7 be the oldest known thermal composite supernova remnant, but owing to its optically revealed cool, dense shell, this remnant supports the proposal that thermal composite supernova remnants lack X-ray bright shells because they have evolved beyond the adiabatic phase. These observations also reveal a slightly extended point source centered on $\mathrm{RA}=19^{\mathrm{h}} 36^{\mathrm{m}} 46^{\mathrm{s}}$, dec $=30^{\circ} 40^{\prime} 07^{\prime \prime}$ and extending 6.5 arcmin in radius in the band 67 map. The source of this emission has yet to be discovered, as there is no known pulsar at this location.
\end{abstract}

Subject headings: ISM: individual (G65.2+5.7) - individual (G65.3+5.7) - supernova remnants - X-rays:ISM 


\section{Introduction}

Within a decade of being identified as a supernova remnant (SNR), G65.2+5.7 (also called $\mathrm{G65.3+5.7)}$ was the subject of several observational papers published in quick succession. However, once its radio and optical images and its optical and X-ray spectra were recorded, the remnant was ignored. Now, a quarter of a century later, G65.2+5.7 has once again become interesting, this time because it helps to explain an unusual class of supernova remnants called thermal composite SNRs (also known as mixed morphology SNRs).

Both of these terms describe the combination of a shell-like radio continuum morphology with a centrally-bright X-ray morphology in which the X-ray emission is due to thermal, rather than synchrotron, processes. Compared with shell-type and plerionic SNRs, these remnants have more mysterious origins. Various workers have suggested that thermal composite SNRs lack X-ray shells because they have evolved into the radiative phase (and thus have cool, X-ray dim shells), or that their centers are X-ray bright because thermal conduction, other forms of entropy mixing, or cloudlet evaporation has enhanced the central densities. Ejecta enrichment or dust destruction could increase the metallicities in the centers, also enhancing the X-ray emission. Alternatively, a thermal composite morphology may be due to collisions with molecular clouds. Interested readers are directed to White \& Long (1991), Long et al. (1991), Cox et al. (1999), Shelton et al. (1999), Chevalier (1999), Yokogawa et al. (2002), Kawasaki (2003), and Shelton, Kuntz \& Petre (2004) for additional discussions of these processes.

Currently, there are few observations of the "smoking gun" for the radiative phase evolution hypothesis: H I shells. The H I shell on W44 is the only possible example (Koo \& Heiles (1995), with interpretations in Cox et al. (1999) and Shelton et al. (1999)). Here we demonstrate that another thermal composite supernova remnant, G65.2+5.7, is clearly in the radiative phase. Ironically, this demonstration is not performed by discovering that a previously known thermal composite SNR is in the radiative phase, but is done by demonstrating that a previously known radiative phase SNR is a thermal composite. In Section 2, we discuss published optical observations, indicating that G65.2+5.7 has entered the shell formation phase; we also discuss published radio and low spatial resolution X-ray observations, showing that G65.2+5.7 has a shell-like radio continuum morphology and emits thermal X-rays. With these characteristics, G65.2+5.7 meets two of the three requirements for the thermal composite classification. The third requirement, that the remnant has a centrally filled X-ray morphology, is established in Section 3, through the analysis of archival ROSAT observations. Furthermore, model fits to the ROSAT data imply that the temperature peaks in the center and decreases mildly with radius, as is found for other thermal composite supernova remnants. In Section 4 , we summarize the results. 


\section{Previous Observations}

The first observations of G65.2+5.7, those made with the red prints of the Palomar Sky Survey (Sharpless 1953), only found two bright filaments, S91 and S94, but failed to recognize the entire outline of the remnant. The true extent of the remnant remained unknown until Gull, Kirshner \& Parker (1977)'s optical emission line survey of the Galactic plane revealed filaments circumscribing a $4^{\circ} \times 3.3^{\circ}$ ellipse. Reich, Berkhuijsen \& Sofue (1979)'s radio frequency data also suggested a similarly sized shell-type remnant.

Even before its true extent was revealed, Sabbadin \& D'Odorico (1976) used their $\mathrm{H} \alpha$, $\left[\mathrm{N}_{\mathrm{II}}\right]$, [S II] $(6717 \AA)$, and [S II] $(6731 \AA)$ measurements to show that the S91 and S94 filaments were dense $\left(n>450 \mathrm{~cm}^{-3}\right)$ shell fragments belonging to a large supernova remnant. Fesen, Blair \& Kirshner (1985) made additional optical emission line measurements of the $\mathrm{S} 91$ filament and of one of the brightest $\left[\mathrm{O}_{\mathrm{III}}\right]$ filaments. Both regions have large $[\mathrm{OI}] / \mathrm{H} \beta$ ratios, which, when compared with the shock models of Raymond et al. (1988), indicate that the photons were emitted by thick $\left(N_{H}>10^{18.3} \mathrm{~cm}^{-2}\right)$ postshock cooling regions behind moderate velocity $\left(v_{s}=100\right.$ to $\left.120 \mathrm{~km} \mathrm{~s}^{-1}\right)$ shocks. Given its [OI] $/ \mathrm{H} \beta$ ratio, S91's relatively modest $\left[\mathrm{O}_{\mathrm{III}}\right] / \mathrm{H} \beta$ ratio indicates that the cooling and recombination behind the shock is "complete" (Raymond et al. 1988). The cooling zone along Fesen, Blair \& Kirshner (1985)'s second pointing direction appears to be well developed, but incomplete. Given that the second pointing direction was chosen for its anomalously large $\left[\mathrm{O}_{I I I}\right] / \mathrm{H} \beta$ ratio, this portion may be less complete than most of the remnant's shell. Similarly, Mavromatakis et al. (2002) found regions of incomplete cooling as well as regions of nearly complete cooling. The shell temperature found from the older $\left[\mathrm{O}_{\mathrm{III}}\right]$ and $[\mathrm{S} \mathrm{II}]$ measurements is $\sim 38,000 \mathrm{~K}$ (Fesen, Blair \& Kirshner 1985; Sabbadin \& D'Odorico 1976). Mavromatakis et al. (2002) calculated shock velocities of 90 to $140 \mathrm{~km} \mathrm{sec}^{-1}$, which are roughly consistent with Rosado (1981)'s kinematically determined expansion velocity of $90 \pm 30 \mathrm{~km} \mathrm{~s}^{-1}$. In summary, G65.2+5.7 is sufficiently evolved for the shock to be weak and the gas behind it to be relatively cool and recombined.

Multiple techniques have been employed to estimate the remnant's size and distance. Reich, Berkhuijsen \& Sofue (1979) applied the $\Sigma$-D relation to their $1420 \mathrm{MHz}$ data obtaining a diameter of $75_{-25}^{+50} \mathrm{pc}$ and a distance of $900_{-300}^{+600} \mathrm{pc}$. Lozinskaya (1981) applied the Galactic kinematic relationship to her Fabry-Perot interferometric $\mathrm{H} \alpha$ data in order to estimate the distance as $800 \mathrm{pc}$ and the minor and major axes of this elliptically shaped remnant as 56 and $64 \mathrm{pc}$, respectively. With a distance of $900_{-300}^{+600} \mathrm{pc}$ and Galactic latitude of $5.7^{\circ}$, the 
remnant lies $90_{-30}^{+60} \mathrm{pc}$ above the Galactic disk. Furthermore, G65.2+5.7's age has been estimated as $\sim 3 \times 10^{5}$ yr (Gull, Kirshner \& Parker (1977), using radiative phase SNR relations).

While others were observing the remnant with optical and radio-frequency detectors, Snyder et al. (1978) and Mason et al. (1979) were observing it with X-ray detectors. Mason et al. (1979)'s HEAO 1 (bandpass $=0.2$ to $2.5 \mathrm{keV}, \mathrm{FWHM}=3^{\circ}$ ) spectrum exhibits line emission, the hallmark of thermal emission from hot plasma. The model which best fits the HEAO 1 spectrum is a Raymond and Smith thermal model with a temperature between 2.0 and $3.6 \times 10^{6} \mathrm{~K}$ (Mason et al. 1979). Although they were not able to resolve the emission spatially with the $H E A O 1$ data, they were able to show that the emission region is not point-like. In the following section, we extend upon this work. We use archival ROSAT data to map the remnant, confirming that the emitting gas is extended and finding that it fills the remnant's outline, lacks a bright shell, and exhibits a slowly decreasing temperature gradient.

\section{ROSAT PSPC X-Ray Observations}

Between August 1995 and September 1997, the remnant was mapped with a series of overlapping ROSAT PSPC observations. We processed the ROSAT PSPC data (removed the contamination, scattered solar X-rays, long-term enhancements, after-pulses, particle background, and bright point sources) and mosaiced the pointings according to the extended object analysis procedures described in Snowden \& Kuntz (1998) and Kuntz \& Snowden (1998). The resulting images in the ROSAT 1L2 band (sensitive to 0.11 to $0.284 \mathrm{keV}$ photons), 45 band (sensitive to 0.44 to $1.21 \mathrm{keV}$ photons), and 67 band (sensitive to 0.73 to 2.04 $\mathrm{keV}$ photons) are displayed in Figure 1, along with a 1L2 band image overlayed with contours from the 67 band map. With the exception of a ridge of band 1L2 emission along the northeast edge and roughly coincident with bright optical filaments, the periphery is X-ray dim. Most of the projected interior shows X-ray emission. Because of its generally "centrally filled" X-ray morphology, thermal X-ray emission, and "shell-type" radio continuum morphology, the remnant should now be classified as a thermal composite.

Comparison of the soft and hard band images shows that the X-ray emission originating in the SNR interior is slightly harder than that originating nearer to the edges. We quantify this trend with a spectral model. The gas is assumed to be near collisional ionizational equilibrium, as is observed both in similar remnants such as W44 (Harrus et al. 1997; Shelton, Kuntz \& Petre 2004), 3C391 (Chen \& Slane 2001), and 0045-734 in the SMC (Yokogawa et al 2002), and in simulated remnants which have evolved long enough to have 
centrally-peaked X-ray morphologies (Shelton 1999). Given ROSAT's spectral resolution, the differences between equilibrium and nearly equilibrium spectra are inconsequential, as are differences between newer and older versions of the Raymond \& Smith (1977) spectral code. In general, the model spectra are calculated and handled according to the techniques described in Kuntz \& Snowden (2000). Thus, we used the Raymond \& Smith (1977) spectral code to create a suite of collisional ionizational equilibrium spectral models. The electron temperature $\left(T_{e}\right)$ ranged from $10^{5} \mathrm{~K}$ to $10^{8} \mathrm{~K}$, with increments in $\log \left(T_{e}\right)$ of 0.05 . In order to account for the absorption by the interstellar medium, we multiplied our suite of model spectra by $e^{-\tau}$, where $\tau$ is the product of the absorption column density $\left(N_{H}\right)$ and the absorption cross section (Morrison \& McCammon 1983). $N_{H}$ ranged from $10^{16} \mathrm{~cm}^{-2}$ to $10^{24}$ $\mathrm{cm}^{-2}$, with increments in $\log \left(N_{H}\right)$ of 0.10 . The resulting spectra were convolved with the ROSAT PSPC response matrix, yielding the countrates in each ROSAT PSPC band.

We prepared the data for comparison with the calculated models by excluding counts from regions of the X-ray image that were contaminated by bright point sources or dominated by the Galactic background emission, dividing the the remaining SNR area into ten, concentric, nearly equal area annuli. These annuli were centered on RA $=19^{\mathrm{h}} 33^{\mathrm{m}} 41^{\mathrm{s}}$, dec $=31^{\circ} 15^{\prime} 57^{\prime \prime}$ in J2000 coordinates and had outer radii of $26,39,48,56,64,71,79,86,94$, and 122 arcmin, respectively. Regions beyond the SNR and regions contaminated by point sources (including the slightly extended hard source centered on $\mathrm{RA}=19^{\mathrm{h}} 36^{\mathrm{m}} 46^{\mathrm{s}}$, dec $=$ $\left.30^{\circ} 40^{\prime} 07^{\prime \prime}\right)$ were excluded from the annuli areas. As a result, in order to create approximately equal area masks, the outermost annuli were extended to relatively large radii. We then calculated the countrates in each of the ROSAT PSPC bands for each annulus. The background countrate for each band was calculated from the cleaned data for the portion of the observed sky that lies outside the SNR's footprint and subtracted from the countrate for each annulus, resulting in the "observed countrates". The standard chi-squared test was used to compare the observed countrates in the ROSAT $1 \mathrm{~L}, 4,5$, and 6 bands for each annulus with the countrates predicted for the spectral models. Band 2 was omitted, owing to complications of an absorption edge in the detector and band 7 was omitted owing to its low countrate. The best fitting models were then selected for each annulus. Figure 3 displays the observed and model spectra for the seventh annulus (spanning the region between 79 and 86 arcmin from the remnant's center).

The best-fit temperatures range from 2.5 to $3.5 \times 10^{6} \mathrm{~K}$ and decrease slightly with radius (see Figure 2). The shallow temperature gradient is a common feature of thermal composite SNRs (i.e., 3C400.2 (Yoshita et al. 2001), Kes 27 (Enoguchi et al. 2002), W44 (Shelton, Kuntz \& Petre 2004)). In the best fit models for the various regions, the column densities ranged from $8 \times 10^{19}$ to $1.3 \times 10^{20} \mathrm{~cm}^{-2}$, but did not exhibit a radial trend. 
The SNR's apparent flux of 0.11 to $2.04 \mathrm{keV}$ photons is $6.8 \times 10^{-11} \mathrm{ergs} \mathrm{cm}^{-2} \mathrm{~s}^{-1}$. If there had been no interstellar scattering, the flux would have been $8.4 \times 10^{-11} \mathrm{ergs} \mathrm{cm}^{-2} \mathrm{~s}^{-1}$. Assuming that the remnant is $900 \mathrm{pc}$ distant, its luminosity is $8.2 \times 10^{33} \mathrm{ergs} \mathrm{s}^{-1}$ and the electron density of the X-ray emitting gas is $0.013 \mathrm{~cm}^{-3}$. Based on the theoretical predictions and hydrocode simulations of the archetypical thermal composite supernova remnant, W44 (Cox et al. (1999), Shelton et al. (1999)), we expect the ambient density to be about 10 times the density within the remnant's center. Thus, the ambient density would be roughly $0.1 \mathrm{~cm}^{-3}$, a reasonable value at G65.2+5.7's location $90 \mathrm{pc}$ above the Galactic plane. The electron density is lower than that of W44, causing the luminosity to be lower as well.

In addition to displaying a centrally concentrated SNR morphology, the band 45 and band 67 X-ray images also reveal a "slightly extended point source" centered on $\mathrm{RA}=19^{\mathrm{h}}$ $36^{\mathrm{m}} 46^{\mathrm{s}}$, dec $=30^{\circ} 40^{\prime} 07^{\prime \prime}$ and extending approximately 6.5 arcmin in radius in the harder band map and less far in the band 45 map. The 1L2 band map does not show excess emission at or near this location. The observed spectrum is too noisy for meaningful fitting to model spectra and there is no known pulsar at this location. Although the Princeton-Arecibo survey for millisecond pulsar's did not find a pulsar coincident with the observed bright spot, it did find a pulsar, J1931+30, within the remnant's outline (Camilo et al. 1996). We did not find excess X-ray emission at its location, $\mathrm{RA}=19^{\mathrm{h}} 36^{\mathrm{m}} 46^{\mathrm{s}}$, $\operatorname{dec}=30^{\circ} 40^{\prime} 07^{\prime \prime}$.

\section{Conclusion}

These ROSAT observations, in combination with existing radio observations, establish G65.2+5.7's membership in the thermal composite (mixed morphology) class of supernova remnants. The radio continuum shells and thermal X-ray bright centers of this puzzling class of remnants have inspired several theoretical explanations. In one scenario, these remnants have evolved beyond the adiabatic phase into the radiative phase. As a result, their peripheries are now cool and not X-ray emissive. This scenario can explain why these remnants lack X-ray bright shells, while other explanations are required in order to account for the extreme brightnesses of these remnants' centers. Here, we note that crucial support for the evolutionary hypothesis is provided by optical evidence of nearly complete cooling behind G65.2+5.7's relatively slow shockfront.

If Gull, Kirshner \& Parker (1977)'s age estimate is correct, then G65.2+5.7 is the oldest known thermal composite supernova remnant. The fact that several other thermal composite supernova remnants neighbor molecular clouds has led to the suggestion that collisions with molecular clouds cause the characteristic thermal composite morphology. In order to 
test this suggestion on G65.2+5.7, we searched the Dame et al. (2001) CO survey data and the Hartmann \& Burton (1997) H I survey data near $l=65.2^{\circ}, b=5.7^{\circ}$ at the remnant's systemic velocity $\left(+8 \pm 10 \mathrm{~km} \mathrm{~s}^{-1}\right.$, Lozinskaya (1981)). Confusion with Galactic material made the search inconclusive. The possibility of a SNR-cloud interaction could potentially be addressed with a targeted search for $\mathrm{CO}$ emission features outlining the remnant.

\section{Acknowledgements}

R.L.S. wishes to thank the National Research Council and NASA's Long Term Space Astrophysics Grant (NAG5-10807) for financial support and the anonomous referee for his/her helpful comments. K.D.K. wishes to thank GSRP for financial support. This research has made use of data obtained from the High Energy Astrophysics Science Archive Research Center (HEASARC), provided by NASA's Goddard Space Flight Center. 


\section{REFERENCES}

Camilo, F., Nice, D. J., Shrauner, J. A. \& Taylor, J. H. 1996, ApJ, 469, 819

Chen, Y. \& Slane, P. O. 2001, ApJ, 563, 202

Chevalier, R. A. 1999, ApJ, 511, 798

Cox, D. P., Shelton, R. L., Maciejewski, W., Smith, R.'K., Plewa, T., Pawl, A. \& Rozyczka, M. 1999, ApJ, 524, 179

Dame, T. M., Hartmann, D., \& Thaddeus, P. 2001, ApJ, 547, 792

Enoguchi, H., Tsunemi, H., Miyata, E., Yoshita, K. 2002, PASP, 54, 229

Fesen, R. A., Blair, W. P. \& Kirshner, R. P. 1985, ApJ, 292, 29

Gull, T. R., Kirshner, R. P. \& Parker, R. A. R., 1977, ApJ, 215, L69

Harrus, I. M., Hughes, J. P., Singh, K. P., Koyama, K., Asaoka, I. 1997, ApJ, 488, 781

Hartmann, D., \& Burton, W. B. 1997, Atlas of Galactic Neutral Hydrogen (Cambridge: Cambridge Univ. Press)

Kawasaki, M. T. 2003, Institute of Space and Astronautical Science Research Note, 764 (PhD Thesis)

Koo, B.-C. \& Heiles, C. 1995, ApJ, 442, 679

Kuntz, K. D. \& Snowden, S. L. 1998, "Cookbook for Analysis Procedures for ROSAT XRT Observations of Extended Objects and Diffuse Background, Part II: Mosaics"

Kuntz, K. D. \& Snowden, S. L. 2000 ApJ, 543, 195

Long, K. S., Blair, W. P., White, R. L. \& Matsui, Y. 1991, ApJ, 373, 567

Lozinskaya, T. A. 1981, Sov. Astron. Lett., 7, 17

Mason, K. O., Kahn, S. M., Charles, P. A., Lampton, M. L. \& Blissett, R. 1979, ApJ, 230, L163

Mavromatakis, F., Boumis, P., Papamastorakis, J. \& Ventura, J. 2002, A \& A, 388, 355

Morrison, R. \& McCammon, D. 1983, ApJ, 270, 119 
Raymond, J. C., Hester, J. J., Cox, D., Blair, W. P., Fesen, R. A. \& Gull, T. R. 1988, ApJ, 324,869

Raymond, J. C. \& Smith, B. W. 1977, ApJS, 35, 419

Reich, W., Berkhuijsen, E. M. \& Sofue, Y. 1979, A \& A, 72, 270

Rosado, M. 1981, ApJ, 250, 222

Sabbadin, F. \& D'Odorico, S. 1976, A \& A, 49, 119

Sharpless, S. 1953, ApJ, 118, 362

Shelton, R. L., Kuntz, K. D. \& Petre, R. 2004, Ap.J, in press, v611

Shelton, R. L. 1999, ApJ, 521, 217

Shelton, R. L., Cox, D. P., Maciejewski, W., Smith, R. K., Plewa, T., Pawl, A. \& Rozyczka, M. 1999, ApJ, 524, 192

Snowden, S. L. \& Kuntz, K. D. 1998, "Cookbook for Analysis Procedures for ROSAT XRT Observations of Extended Objects and Diffuse Background, Part I: Individual Observations"

Snowden, S. L., McCammon, D., Burrows, D. N. \& Mendenhall, J. A. 1994, ApJ, 424, 714

Snyder, W. Davidsen, A. F., Henry, R. C., Shulman, S., Fritz, G. \& Friedman, H. 1978, ApJ, 222, L13

White, R. L. \& Long, K. S. 1991, ApJ, 373, 543

Yokogawa, J., Imanishi, K., Koyami, K., Nishiuchi, M. \& Mizuno, N. 2002, PASJ, 54, 53

Yoshita, K., Tsunemi, H., Miyata, E., Mori, K. 2001, PASJ, 53, 93 


$$
-10-
$$

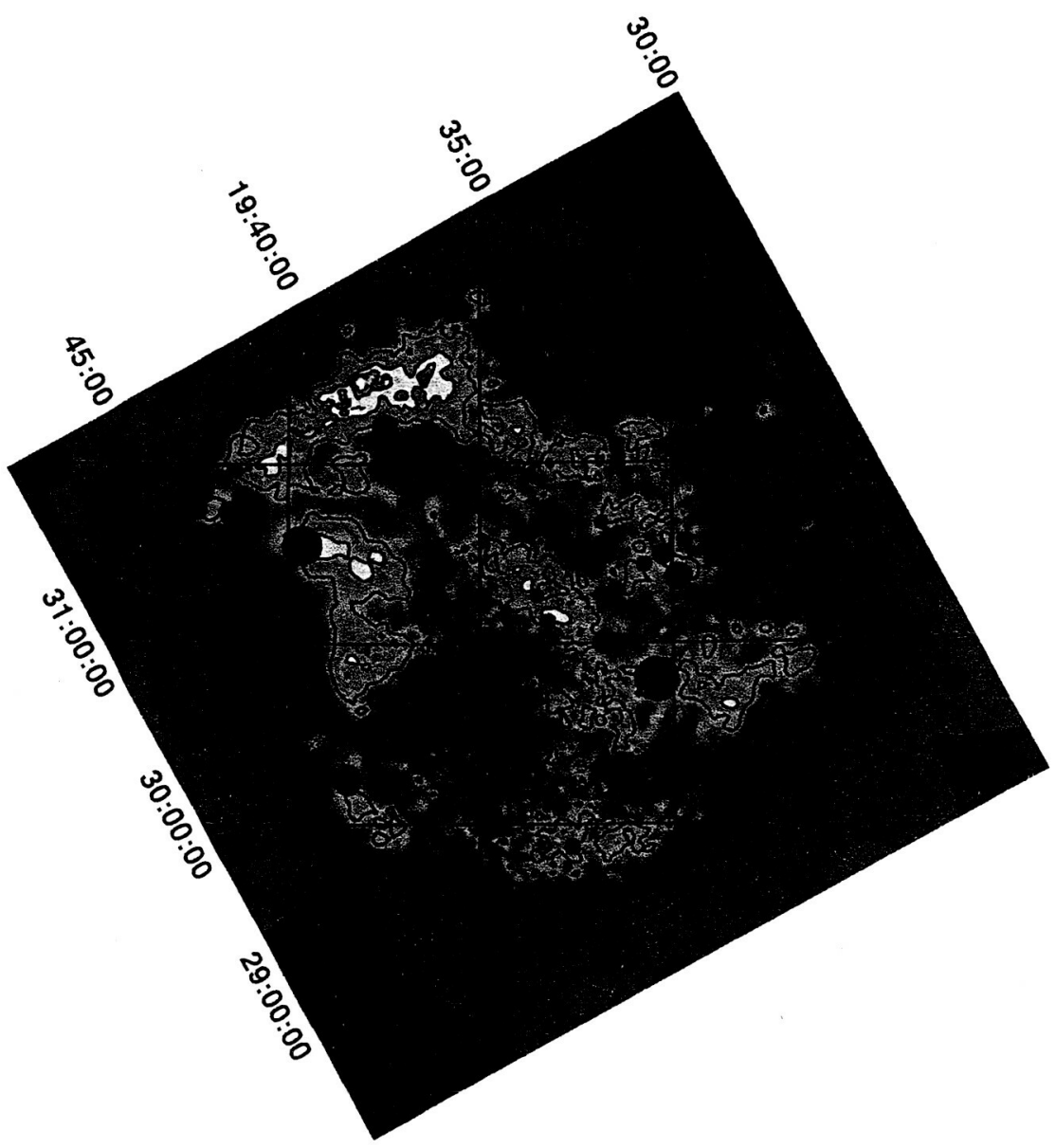




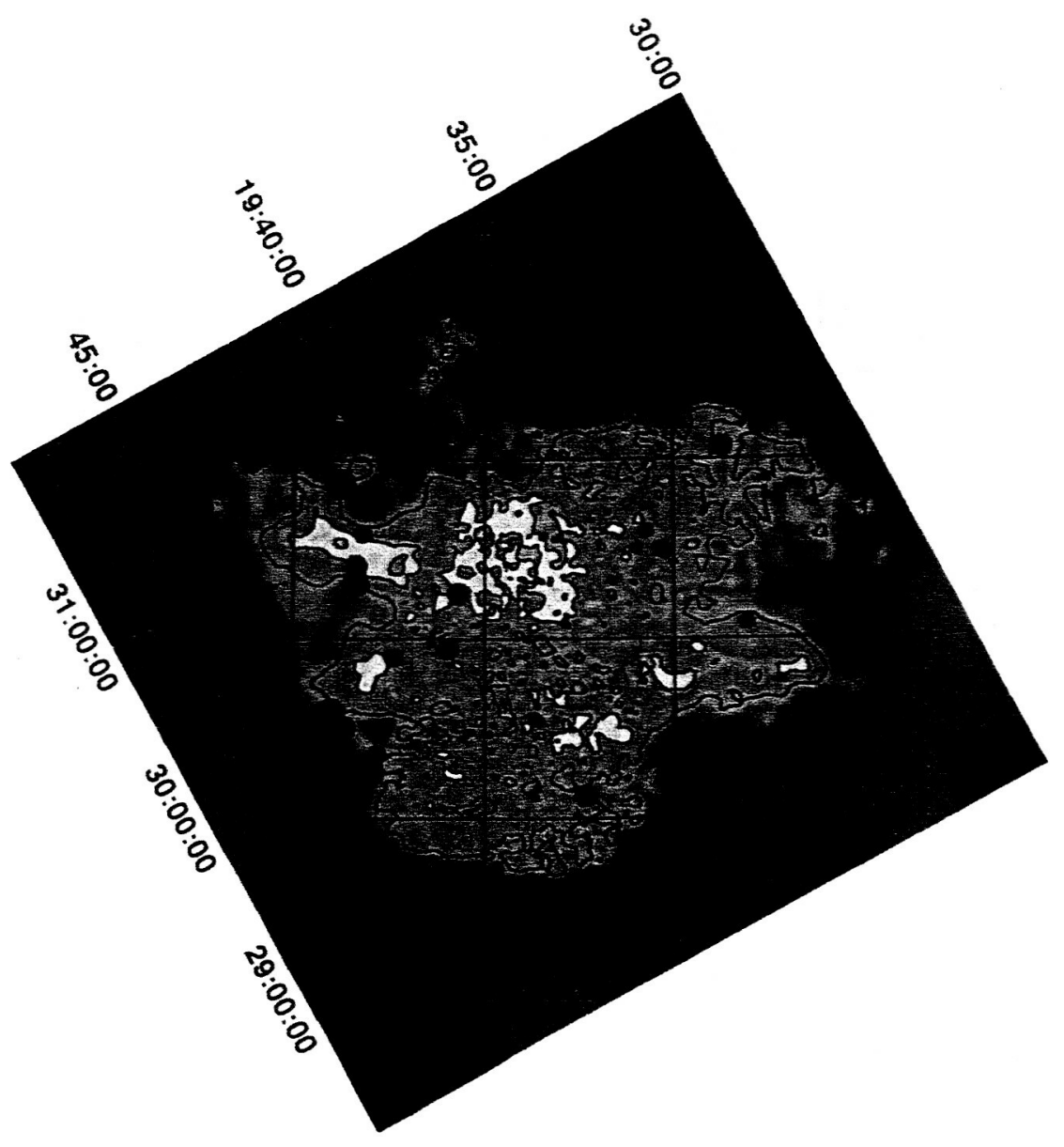




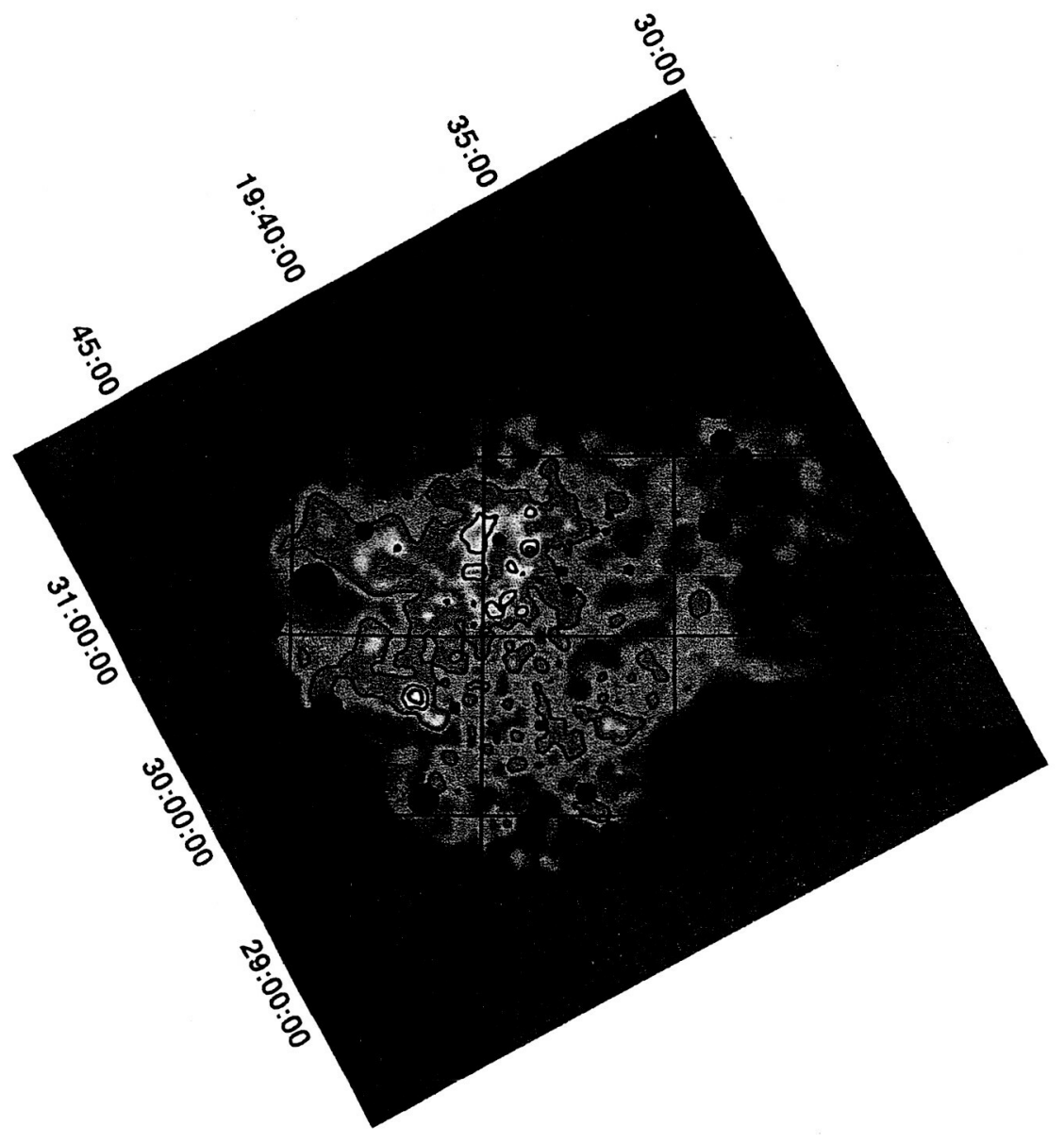




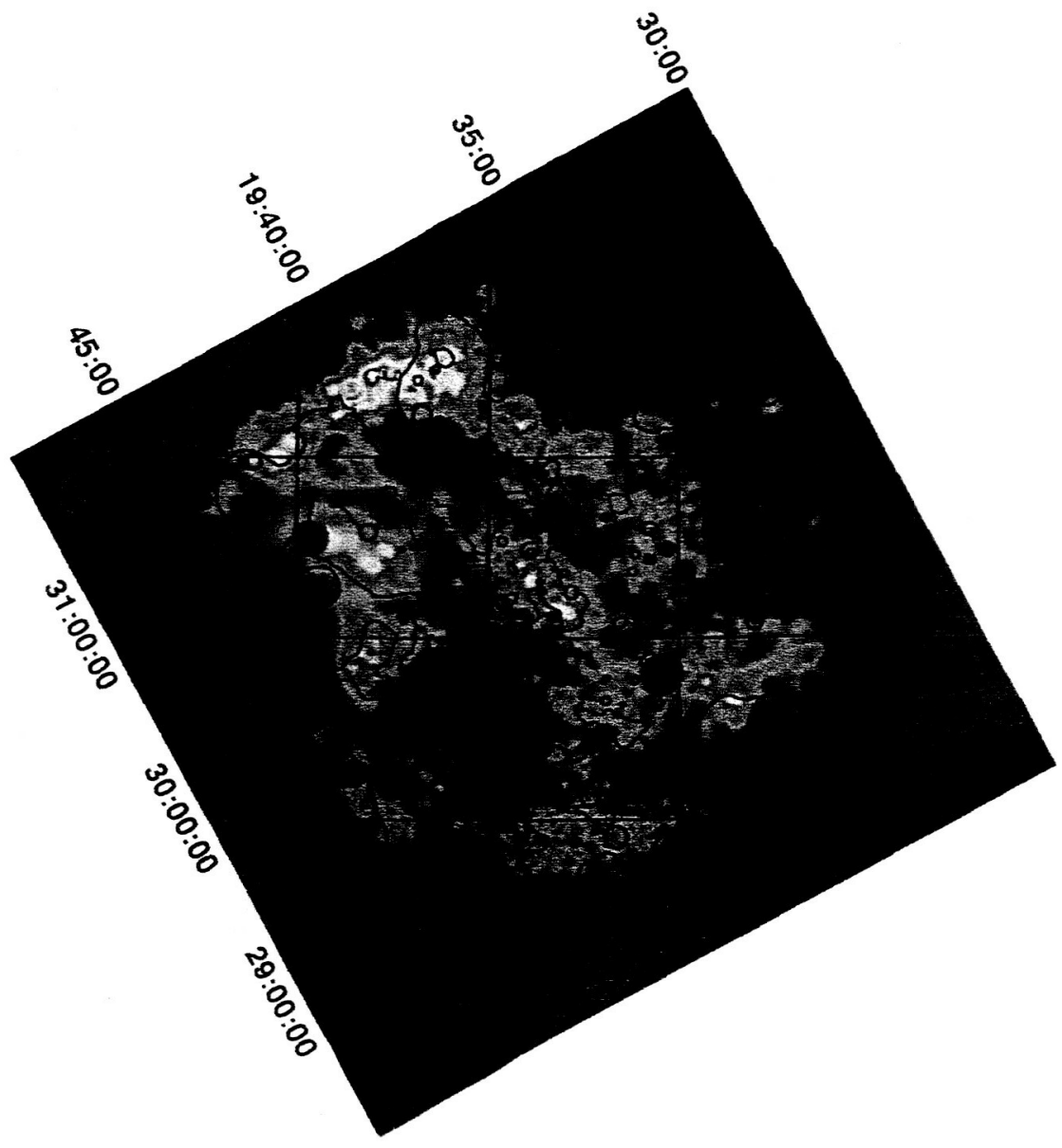

Fig. 1.- Mosaic maps of G65.2+5.7 in J2000 coordinates. The black disks show where bright point sources have been removed. Top: Map in the ROSAT 1L2 band, made from PI channels 11 to 41 and sensitive to 0.11 to $0.284 \mathrm{keV}$ radiation. Contours are drawn at 125 , 250,375 , and $500 \times 10^{-6}$ counts $\mathrm{s}^{-1}$ arcmin $^{-2}$. Second: Map in the ROSAT 45 band, made from PI channels 52 to 90 and sensitive to 0.44 to $1.21 \mathrm{keV}$ radiation. Contours are drawn at $75,150,225$, and $300 \times 10^{-6}$ counts $\mathrm{s}^{-1} \operatorname{arcmin}^{-2}$. Third: Map in the ROSAT 67 band, made from PI channels 91 to 201 and sensitive to 0.73 to $2.04 \mathrm{keV}$ radiation. Contours are drawn at 50,100, 150, and $200 \times 10^{-6}$ counts $\mathrm{s}^{-1}$ arcmin $^{-2}$. Bottom: Map in the ROSAT 1L2 band, overlayed with band 67 contours. The semi-circle contour in the lower left of the image is an artifact of the pointsource removal in the band 67 map. These X-ray images reveal G65.2+5.7's "center filled" morphology as well as its slight spectral hardness gradient. 


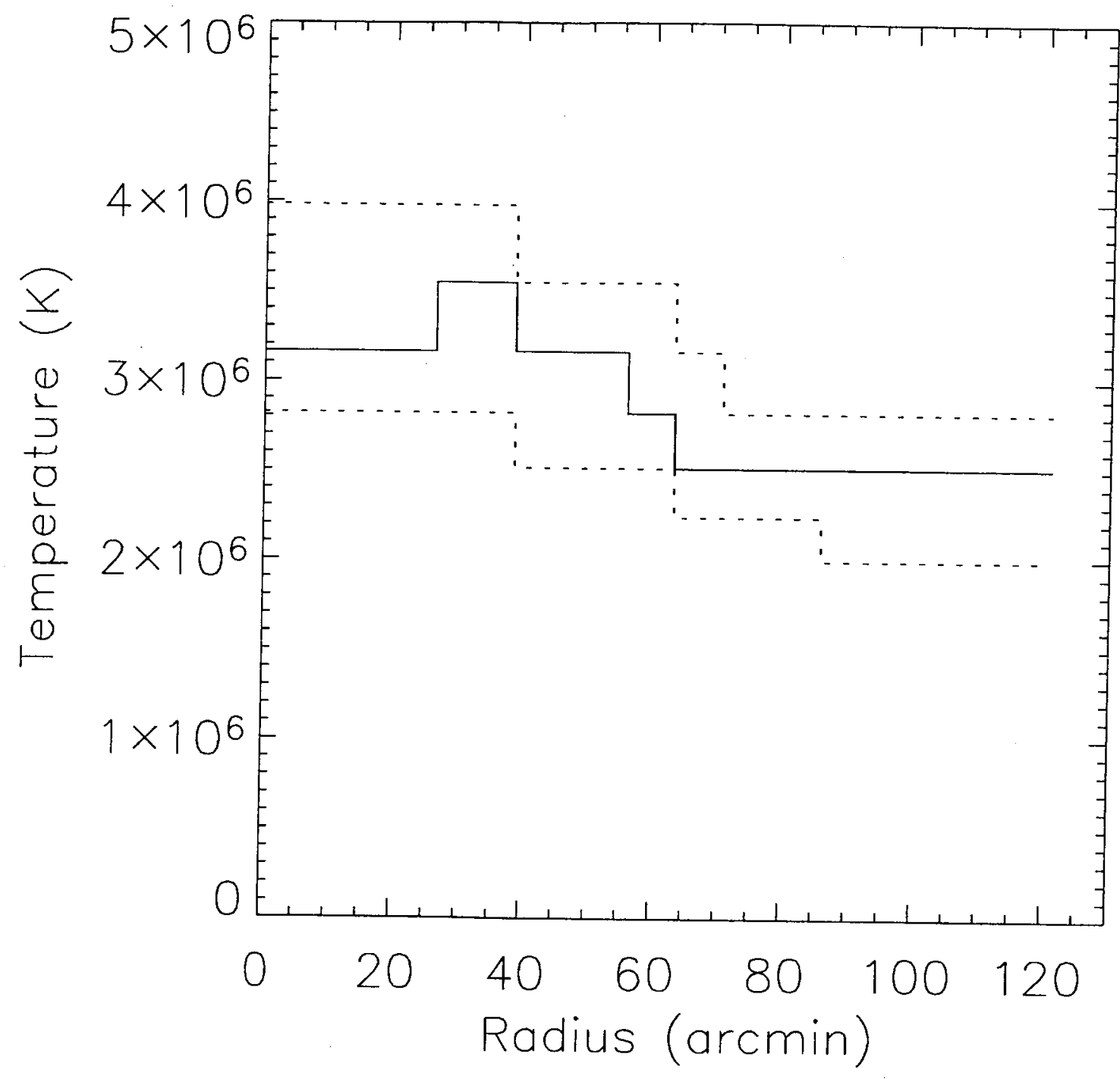

Fig. 2. - The solid histogram traces the electron temperature of the best fitting plasma model as a function of radius. The dotted histograms trace the $\pm 1 \sigma$ values. 


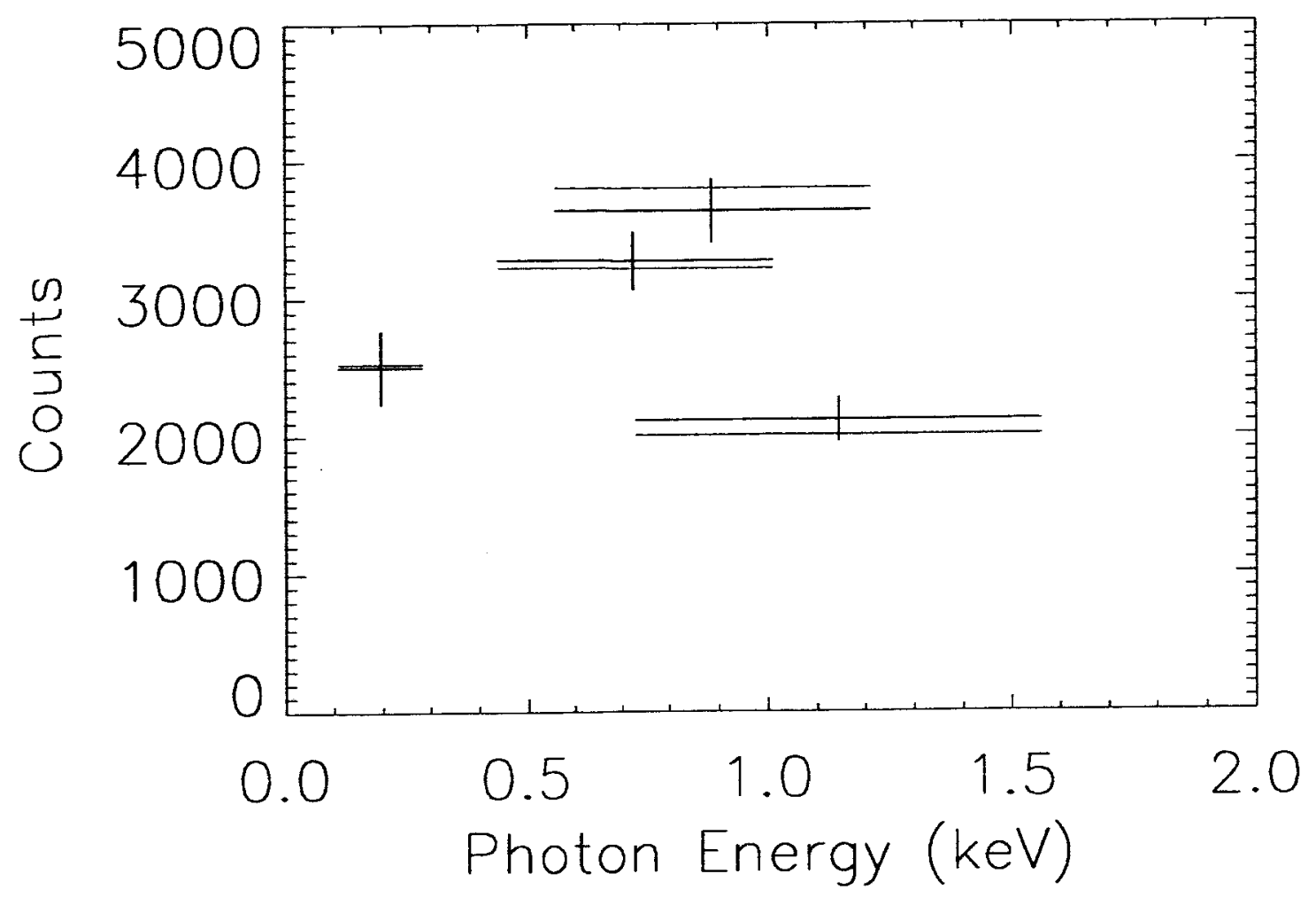

Fig. 3. - Observed (black) and model (red) spectra for the seventh annulus. The $1 \sigma$ error bars were calculated from the pixel to pixel variation in the observational data. Band 2 data have been ignored owing to complications with an absorption edge in the detector, while band 7 data have been ignored owing to the low countrate. The ROSAT PSPC band photon energies were taken from Snowden et al. (1994). This fit has $\chi^{2}=1.1$ with 1 degree of freedom and is neither the best nor the worst case among the annular fits. 